SCUltURA IN PEZZI: APPUNTI SUlla SCULTURA ALTO MEDIEVAlE Di SANTA PRASSEDE.

\title{
Antonella Ballardini
}

Università degli studi Roma Tre

e-mail: antonella.ballardini@uniroma3.it

Rebut: 6 febrer 2017 | Revisat: 22 març 2017 | Acceptat: 15 abril 2017 | Publicat: 21 juny 2017 | doi: 10.1344/Svmma2017.9.3

\section{Resum}

Dedicato ai frammenti scultorei della basilica di Santa Prassede (817-824), il contributo propone alcune riflessioni su un episodio emblematico dell'età classica e creativa della scultura alto medievale a Roma.

Paraules clau: scultura alto medievale, arredo liturgico, Santa Prassede, Roma, Oratorio di San Zenone, Pasquale I

\begin{abstract}
This paper looks into the fragments of liturgical furniture of Santa Prassede (817-824): an emblematic example of classical and creative sculpture in the early medieval Rome.
\end{abstract}

Key Words: early medieval sculpture, liturgical furniture, Santa Prassede, Rome, Saint Zeno Chapel, Pascal I 
Per chi si occupi di alto medioevo, i frammenti scultorei della basilica romana di Santa Prassede sono tra i più noti, non solo per la qualità esecutiva che li distingue, ma anche perchè, nell'incerto orizzonte cronologico della scultura di quell'età, si possono inquadrare con discreta sicurezza.

I quattro plutei, quasi integri che, con un modesto numero di frammenti di lastre decorate, si conservano nella basilica dell'Esquilino, presentano infatti caratteristiche tecnico-esecutive e un repertorio decorativo che trovano riscontro nei frammenti scultorei di Santa Maria in Domnica e di Santa Cecilia, rispettivamente la diaconia sul colle Celio e l'antico titulus nel Trastevere che, con Santa Prassede, furono riedificati da papa Pasquale I (817-824). ${ }^{1}$ (fig. 1)

Legata alla figura leggendaria di Prassede «al Signore grata più delle stelle» ${ }^{2}$ e quasi personificazione del fondamento martiriale della Chiesa di Roma, la vicenda millenaria della basilica è indagabile attraverso fonti letterarie ed epigrafiche; documenti d)archivio (antichi e recenti) e una ancorchè parziale indagine archeologica che hanno precocemente attirato l'interesse di eruditi e di studiosi. ${ }^{3}$

Prima che nella basilica tornassero alla luce i quattro plutei frammentari scolpiti in grandi lastre di marmo cipollino (1915), già Raffaele Cattaneo nel volume L'architettura in Italia dal VI secolo al Mille (1889) segnalava il celebre l'oratorio di San Zenone come «la cosa più ragguardevole che racchiuda Santa Prassede fra i resti del secolo IX». ${ }^{4}$ È probabile che il giudizio dello studioso fosse indotto dal contesto d'insieme - quello della basilica negli anni '80 del XIX secolo - che egli vedeva alterato dagli interventi decorativi "in stile Pio IX". 5

Tuttavia, se riletto nella prospettiva metodologica che ispira L'Architettura in Italia, l'accenno al San Zenone lascia intendere come per Cattaneo l'oratorio fosse «ragguardevole» in sè e ai fini della ricomposizione di un più generale quadro diacronico dell'architettura e della scultura nell'alto medioevo: San Zenone infatti non solo conservava un'iscrizione «sincrona» ${ }^{6}$, ma soprattutto mostrava inalterato (e mostra ancora oggi) l'originario vincolo «organico» tra la sua scultura architettonica e il resto dell'edificio. ${ }^{7}$

\footnotetext{
${ }^{1}$ Pani Ermini 1974a: 111-113, Tavv. XXII-XXXIV; Melucco Vaccaro 1974: 167-175, Tavv. XLIX-LII; Ranucci 2003: 218-227 RighETTI 2007: 78-81; BALLARDINI 2008: 225-246 e per un esame tecnico delle sculture MACCHIARELLA 1976a: 282-288.

${ }^{2}$ «...Praxedis D(omi)no super aethra placentis...», come si legge nell'iscrizione musiva dell'abside della basilica, v. FaVReau 1992 pp. 681-727; FaVReau 1997, pp. 114-120.

${ }^{3}$ Ciampini 1699: 143-143; Davanzati 1725; Krautheimer, Corbett 1971: 235-262; Apollonj Ghetti 1961; Ballardini 1999: 5-68; Caperna 1999; per un riesame della documentazione di scavo di B.M. Apollonj Ghetti, v. da ultimo CAPERNA 2014: 51-57 e 79-80.

${ }^{4}$ Cattaneo 1889: 153; per un profilo biografico di Raffaele Cattaneo v. Russo: 2012: 291-292.

${ }^{5}$ «Putroppo goffi restauratori moderni hanno guasto linterno di questa basilica, coprendone le pareti con volgari e stonate pitture...», CATTANEO 1889: 152; CAPERnA 2014: 38-39.

${ }^{6}$ Paschalis praesulis opus decor fulgit in aula / quod pia optulit vota studuit reddere D(omino)o: «Di Paschal l'opra in questa reggia splende / Che i voti a Dio, c'havea promessi, rende», v. PAnvinio 1570: 333 (traduzione di M. A. Lanfranchi).

${ }^{7}$ Come per Pietro Selvatico, anche per Raffaele Cattaneo scrivere di architettura comportava necessariamente scrivere di scultura architettonica, BALLARDINI 2009: 109-115; BALLARDINI 2013: 159.
} 
Con un occhio affinato dalla formazione classicista e dalle competenze tecniche di architetto, lo studioso fu in grado di stabilire la cronologia relativa dell'apparato scultoreo-decorativo del sacello, pavimento compreso, che egli riconobbe tra i più antichi esempi medievali di opus sectile. ${ }^{8}$ In San Zenone, Cattaneo individuò i reimpieghi di età classica rilavorati nel IX secolo e, riconoscendo alcuni elementi alto medievali di riutilizzo, aprì un'inedita prospettiva tanto allo studio della pratica del reimpiego quanto alla nostra conoscenza dello sviluppo della scultura ad intreccio. ${ }^{9}$ (fig. 2) È così che tra le «varie età» palesate dalla scultura architettonica del sacello egli ragiona sulla cronologia dei plinti con basi di colonna in opera all'interno dell'oratorio e richiama l'attenzione sull'adattamento dei capitelli ionici del prospetto esterno - di «mano bizantina» - che distingue dai pulvini decorati a zig-zag, a suo parere dello stesso artefice «italo-bizantino» che scolpì gli stipiti.

Se il quadro classificatorio dello "stile" della scultura alto medievale proposto dallo studioso nell'ultimo quarto del XIX secolo (stile "latino-barbaro"; "bizantino-barbaro"; "italo-bizantino") è da considerare criticamente in una prospettiva storiografica, è un fatto però come ancora di recente si sia ricorsi alla lucida lettura del monumento offerta da Cattaneo, confermando per la scultura architettonica di San Zenone la sostanziale validità della cronologia relativa proposta dallo studioso. ${ }^{10}$

\section{Plutei e pavimento}

È un vero peccato che Raffaele Cattaneo (1861-1889), coetaneo di Giacomo Boni (1859-1925), non sia vissuto abbastanza per seguire i lavori sovraintesi da Antonio Muñoz a Santa Prassede. Proprio in veste di soprintendente, tra il 1913 e il 1918, Muñoz giocò infatti un ruolo decisivo nella scelta di conferire un aspetto neocosmatesco al pavimento di Santa Prassede. ${ }^{11}$ È in quell'occasione che, dal pavimento settecentesco, tornarono alla luce i quattro plutei frammentari e altri frammenti scultorei di minori dimensioni che sono oggi affissi alle pareti dell'antico transetto Nord (cappella del Crocifisso). (fig. 3)

Primo editore di questo gruppo omogeneo di sculture fu lo stesso Muñoz in una breve dissertazione presentata, l'anno successivo al ritrovamento, alla Pontificia Accademia Romana di Archeologia: «...rimuovendosi alcune lastre di marmo bianco si è trovato che nel rovescio essi avevano degli ornati del consueto tipo romano dell'VIII-X secolo. Sono evidentemente due plutei della schola cantorum di Pasquale I. Uno di essi misura 2,20 x $1 \mathrm{~m}$ e ha due cerchi con entro rombi; l'altro $2,24 \times 1 \mathrm{~m}$ ha entro figure geometriche, tre croci di cui in epoca più tarda furono scalpellati $\mathrm{i}$ bracci laterali.».12

\footnotetext{
${ }^{8}$ Cattaneo 1889: 154-155; Guidobaldi, Guiglia Guidobaldi 1983: 468-469.

${ }^{9}$ Sul reimpiego in San Zenone, da ultimo Pensabene 2015: 405-408; 415; 858.

${ }^{10}$ PANi Ermini 1974a: 134-144, Tavv. XXXV-XLV.

${ }^{11}$ CAPERNA 2014: 162-166, figg. 182-185.

${ }^{12}$ v. MuÑoz 1918: 119-128; per un profilo dell'attività di Antonio Muñoz che, tra il 1914 e il 1944, fu responsabile a Roma dei principali cantieri di restauro «medievali» v. Bellanca 2002: 136-137 e 321-322.
} 
Purtroppo i lavori del cantiere vennero condotti trascurando di rilevare la posizione dei reperti e le eventuali tracce lasciate dagli ancoraggi dei plutei e dei pilastrini marmorei alla quota del piancito più antico che avrebbero permesso di fondare lo studio dell'assetto liturgico dell'edificio di Pasquale I su dati archeologici. ${ }^{13}$

Sia pure per via indiretta, qualche indicazione sul pavimento e sul recinto presbiteriale alto medievali si ricava dagli scritti di Benigno Davanzati e di Giovanni Marangoni che videro il pavimento alto medievale della basilica prima del suo completo rifacimento, avvenuto nel 1742 a compimento dei restauri promossi dal cardinale Ludovico Pico della Mirandola. ${ }^{14}$

È così che, nel 1744, Giovanni Marangoni, denunciava la dispersione di innumerevoli epigrafi perpetrata durante il rifacimento dell'antico impiantito della basilica, «la quale da san Pascale papa I fu tutta lastricata con iscrizioni sì gentili come cristiane, onde scelti tutti codesti marmi, riempito ne fu l'atrio della medesima chiesa: e quantunque da noi ne fosse pregato il padre Procuratore Generale, che almeno si salvassero tutti quelli che avevano qualche iscrizione, mi fu risposto, che i monaci non potevano operare cosa alcuna, mentre lo scalpellino aveva co' superiori maggiori patteggiato di rinnovare il pavimento, coll'acquisto di tutti que' marmi: onde si rinnovò con mattoni, e le sole guide di lastre di marmo, benchè però alcune poche tavole con alcune iscrizioni siano state lasciate nelle navi laterali». ${ }^{15} \mathrm{La}$ testimonianza di Marangoni, erudito e conoscitore di memorie antiche, induce a ritenere che, fino al 1742, il pavimento di Santa Prassede fosse rimasto, in certa misura, quello alto medievale lastricato con iscrizioni antiche, pagane e cristiane. Già, l'abate Benigno Davanzati, autore nel 1725 di una guida al pellegrino in visita alla basilica, menzionava l'antico pavimento e riferiva di una barriera o di una recinzione presbiteriale, ai suoi tempi già smatellata, della quale riconosceva alcune lastre di pluteo reimpiegate a terra alla base del podio absidale. Accennando all'antica consuetudine di riservare luoghi separati agli uomini e alle donne, Davanzati nomina infatti i cancelli «posti per togliere la confusione, che suole cagionare la moltitudine...; anche [la nostra basilica] aveva prima questo cancellato, o sia muro divisorio come ordinano le tradizioni Apostoliche...e questi cancellati, o divisioni, sono quelle quattro pietre, o marmi scannellati,...nel pavimento avanti gli scalini dell'altare maggiore, le quali anticamente stavano disposte da una parte e l'altra per formare la divisione fra gli uomini e fra le donne». ${ }^{16}$

\footnotetext{
${ }^{13}$ A distanza di anni, sulle pagine dell'Osservatore Romano, il modo di operare del cantiere sovrainteso da Muñoz fu stigmatizzato come un vero «misfatto archeologico», ricordando come fosse stato impedito a Orazio Marucchi di effettuare dei rilevamenti nel cantiere v. A. Lazzarini, Restauri e scavi al Titulus Praxedis (5 settembre 1937), segnalato da CAPERNA 2014: 166.

${ }^{14}$ A Ludovico Pico della Mirandola dobbiamo anche l'attuale sistemazione tardo barocca del podio e della confessione, v. CAPERNA 2014: 143-157.

${ }^{15}$ Marangoni, 1744: 432.

${ }^{16}$ Poco oltre Davanzati torna sull'argomento: «E circa del nominato Matroneo,...questo era un luogo destinato per le donne, e ferrato con pietre, e sebbene al presente non è in essere, vi è però stato come si ricava e conosce da quelle quattro gran pietre scannellate che sono avanti la scalinata del presbiterio, le quali lo formavano, e che poi non praticandosi più nelle chiese la suddetta divisione,...furono levate e per memoria del suddetto Matroneo poste nel pavimento», v. DAVANZATI 1725: 163-164 e 169.
} 
Ma quando i plutei «scannellati» furono usati a parzialmente risarcimento del pavimento che, nel 1725 , era ancora quello di Pasquale I?

È plausibile che le quattro lastre siano state deposte ai piedi del podio in occasione della riconfigurazione dell'area absidale promossa nel 1489 dal cardinale Antoniotto Pallavicini. Questo radicale intervento comportò un avanzamento della fronte del podio, la trasformazione in cappella del braccio Nord del transetto e, verosimilmente, lo smontaggio del recinto presbiteriale che si allungava nella navata ai piedi del podio. ${ }^{17}$

Seguendo l'esempio della dismissione degli antichi recinti presbiteriali già attuata nelle chiese madri dell'Urbe (San Giovanni in Laterano e San Pietro), il cardinale titolare ridusse così in forma moderna lo spazio liturgico di Santa Prassede. ${ }^{18}$

L'intervento spiegherebbe perchè il cardinal Pallavicini avesse parzialmente restaurato il pavimento alto medievale dell'aula «facendolo riunire in miglior forma e porvi nuovi marmi, dove richiedeva il bisogno» ${ }^{19}$

È plausibile, infatti, che i guasti all'antico piancito fossero stati provocati proprio dallo smontaggio dei plutei e dei pilastrini, un'operazione complessa che doveva aver scompaginato il pavimento, almeno in corrispondenza del perimetro della recinzione che per secoli era rimasta ancorata al suolo.

Così ricapitolata $(1489-1504 ; 1725 ; 1742)$, la vicenda del pavimento dimostra la permanenza in luogo dei quattro plutei frammentari di San Prassede e, quand'anche in modo non assoluto, prova la loro pertinenza al pontificato di Pasquale I. ${ }^{20}$ Non solo: la storia del pavimento di Santa Prassede permette di aggiungere qualche nuova considerazione.

I segni di consunzione della superficie delle lastre riscoperte da Muñoz confermano che, sul finire del Quattrocento, i plutei furono messi a terra con decorazione a vista: è probabile che in quell'occasione il braccio orizzontale delle croci che ornavano i plutei fosse scalpellato, in ottemperanza all'antico divieto che interdiva la conculcatio crucis. ${ }^{21} \mathrm{Nel} 1742$ quegli stessi plutei

\footnotetext{
${ }^{17} \mathrm{Al}$ cardinal Pallavicini si deve inoltre la creazione dei "coretti” ai lati del podio che comportò l'occultamento delle sei celebri colonne corinzie entro muri diaframma, v. CAPERNA 2014: 35-36.

${ }^{18}$ Il venir meno dell'antica pratica stazionale della liturgia, a seguito del trasferimento della curia papale ad Avignone e il progressivo imporsi di nuovi ideali formali dello spazio di culto vedono a Roma l'avvio di un processo di "liberazione della navata" già dal secondo quarto del XV secolo: San Giovanni in Laterano e San Pietro persero il loro recinto presbiteriale tra il 1425 e il 1460, v. DE BLAAUw 2006: 25-51.

${ }^{19}$ DaVANZati 1725: p. 508.

${ }^{20}$ L'allestimento dell'arredo liturgico marmoreo in un edificio di culto come Santa Prassede, riedificato dalle fondamenta, richiedeva la messa in opera del recinto, fatto di pesanti lastre di marmo, a cantiere in corso e insieme con il pavimento, a tale proposito si vedano le considerazioni di Roth-Rubi sul montaggio dell'arredo liturgico primario («Grundausstattung») nella chiesa benedettina di San Giovanni di Müstair, v. Rotн-RuBi 2015b (Textband): 169.

${ }^{21}$ NoBLE 2009: 26.
} 
sarebbero stati rifilati ai margini e riutilizzati nuovamente, ma a rovescio, come guide di marmo da alternare alle mattonelle di cotto del nuovo impiantito. Ma come andarono dispersi tutti i pilastrini e i plutei del recinto presbiteriale della basilica di IX secolo che non sono pervenuti?

È probabibile che una parte delle "pietre" alto medievali avessero subito la stessa sorte delle iscrizioni pagane e cristiane lamentata dal Marangoni, ovvero che anche parte dei marmi della recinzione fosse stata ceduta a compenso degli scalpellini che realizzarono il pavimento settecentesco. ${ }^{22}$ Va tuttavia ricordato come il rifacimento del pavimento delle navate avesse seguito di poco la riqualificazione del podio absidale e della sottoposta camera delle reliquie (1729-1734). ${ }^{23}$ In particolare questo intervento comportò lo smontaggio del ciborio e dell'altare cosmatesco, quest'ultimo trasferito nella nuova cappella della cripta con accesso (longitudinale) dalla navata, un intervento che dovette lasciare il segno nel pavimento medievale del podio che richiese infatti un restauro. Con gusto antiquario, si decise di ricompaginare il tappeto policromo medievale. ${ }^{24}$ Non è possibile dire se l'alternanza delle specchiature dei marmi colorati a guide di buon marmo venato risalisse già ai lavori del cardinal Pallavicini (1489-1504) o se fosse invece l'esito del restauro settecentesco, è rilevante però che la tra specchiature cosmatesche se ne distingua una a semplice scacchiera uguale in tutto, per colori e dimensioni dei sectilia, al tessellato che in San Zenone circonda la grande rota porfiretica.(figg. 4-5) Questa osservazione, che dobbiamo a Giuseppe Finocchio, ha permesso di immaginare che il podio di Pasquale I fosse anch'esso ornato di un pregevole pavimento in opus sectile. ${ }^{25}$ L'ipotesi trova un'indiretta conferma nella testimonianza di Raffaele Cattaneo che ebbe modo di vedere, nell'altrettanto pascaliana basilica di Santa Cecilia, il pavimento dell'area presbiteriale prima dei "restauri" del 1900. Della vecchia tribuna egli ricorda infatti «una porzione del pavimento originario, ove si vedono gli stessi motivi di decorazione, le stesse maniere e gli stessi marmi che offre il pavimento della cappella di San Zenone.». ${ }^{26} \mathrm{Al}$ pari delle modalità costruttive, della decorazione musiva $\mathrm{e}$ dei frammenti di scultorei pervenuti da Santa Prassede, da Santa Maria in Domnica e da Santa Cecilia, la puntuale osservazione di Cattaneo conferma che i cantieri delle tre basiliche, riedificate nell'arco di pochi anni da Pasquale I, erano organizzati come un'impresa "a ciclo continuo", in grado di pianificare tanto la raccolta e l'utilizzo di materiali per costruire e per decorare gli edifici, quanto l'avvicendamento delle maestranze specializzate (muratori, carpentieri, fabbri, scultori e marmorai, frescanti, mosaicisti e stuccatori). ${ }^{27}$

\footnotetext{
${ }^{22}$ I pilastrini erano le pietre più facilmente trasferibili; è indicativo che anche a Santa Sabina i pilastrini alto medievali siano andati dispersi con modalità analoghe, v. GIANANDREA 2011: 154-156.

${ }^{23}$ CAPERNA 2014: 146-151.

${ }^{24}$ FINOCCHIO 2010: 305-312.

${ }^{25}$ FinOCCHIO 2010: 310.

${ }^{26}$ Cattaneo 1889: 155. Negli anni ' 80 dell'Ottocento, Cattaneo stava compiendo uno studio sui pavimenti antichi perchè, come architetto, doveva progettare un impiantito di marmi colorati per la cripta di Pio IX al Verano, BALLARDINI 2010: 210.

${ }^{27}$ BALLARDINI 2008: 225-226.
} 
Tornando al podio di Santa Prassede e al suo pavimento, che Antonio Muñoz lasciò intatto, le guide di marmo venato che incorniciano le specchiature di marmi colorati, allo stesso modo delle guide del pavimento dell'aula che restituirono i quattro plutei di Pasquale I, potrebbero celare altri frammenti del perduto recinto presbiteriale di IX secolo, lo suggeriscono l'ampio impiego del marmo cipollino (lo stesso marmo 'greco' dei plutei frammentari), le dimesioni delle guide (che in alcuni casi superano i 2,30 m di lunghezza) e qua e là la presenza di tracce di decori erasi dalla superficie. ${ }^{28}$ (fig.6)

\section{Schemi decorativi, tecnica e standards qualitativi}

Nella marcata oscillazione degli standards qualitativi della scultura alto medievale, i rilievi prodotti nelle officine di Pasquale I raggiungono esiti che, in anni immediatamente successivi, furono mantenuti nel cantiere di Santa Sabina (824-827). ${ }^{29}$ Con i marmi della basilica dell'Aventino - riallestiti nella celebre "schola cantorum" di Antonio Muñoz ${ }^{30}$, i plutei frammentari delle basiliche pascaliane sono infatti esemplari di quella che potremmo definire l'età classica e felicemente creativa della scultura alto medievale a Roma. ${ }^{31}$

Purtroppo, a Santa Prassede, i frammenti scultorei superstiti non sono ispezionabili sul verso perchè immurati. Considerata la vicenda del loro recupero, il lato nascosto dovrebbe tuttavia corrispondere a quello levigato delle guide di marmo del pavimento settecentesco (supra). ${ }^{32}$

I margini originali delle lastre, quando risparmiati, sono molto danneggiati e solo in due di casi la cornice si conserva quasi integra. ${ }^{33}$ (fig. 7) La presenza di tale rifinitura permette di ritenere $\mathrm{i}$ marmi frammentari come parti di plutei di un setto presbiteriale.

\footnotetext{
${ }^{28}$ I quattro plutei frammentari pervenuti raggiungono mediamente la lunghezza di 2,20 m.

${ }^{29}$ Nella basilica dell'Aventino, papa Eugenio II (824-827) si avvalse di una squadra di lapicidi e di scultori che si era formata nei cantieri pascaliani, v. Trinci Cecchelli 1976: 194-224, Tavv. LXXII-LXXXV; da ultimo, seguendo un'ipotesi di Margherita Cecchelli, Manuela Gianandrea ha riconosciuto un frammento di arco con iscrizione, murato nel giardino della chiesa dei Santi Bonifacio e Alessio, come parte del ciborio di Santa Sabina che Gianandrea attribuisce agli scultori di Eugenio II, v. GiANANDREA 2011: 156-159, figg. 5-6. Se la pertinenza alla basilica dell'Aventino è dedotta in modo plausibile dall'iscrizione, mi permetto di osservare che l'ordine del motivo compositivo e la tecnica poco sorvegliata di questo frammento sono incomparabili con quelli che distinguono $\mathrm{i}$ marmi di Eugenio II, è strano cioè che nell'officina del papa la realizzazione del ciborio (se di ciborio si tratta) sia stata affidata allo scultore meno dotato.

${ }^{30}$ Il riallestimento della "schola cantorum", concluso nel 1936, ebbe una lunga gestazione e cambiamenti in corso d'opera, si veda in proposito Bellanca 1999: 13-19; Ghisu, Raimondi 2008: 303-307; BetTi 2008: 151 e nota 13; GiANANDREA 2011: 153-154.

${ }^{31}$ Pani Ermini 1974a: 111-113, Tavv. XXII-XXXIV; al censimento di Letizia Pani Ermini sono da aggiungere i frammenti di un architrave ricomposti nel Settecento nelle pareti laterali del podio absidale, sopra alle celebri colonne corinzie, v. EMERICK 2001, pp. 146-148; e l'architrave del protiro medievale, che per la tipologia dell'ornato e l'impiego del trapano va distinto dagli artefatti realizzati nelle officine di Pasquale I e accostato a prodotti di ascendenza bizantina che pure non mancano anche tra i reimpieghi di San Zenone e altrove nell'Urbe, si veda per es. un frammento di cornice dell'Antiquarium comunale in Pani Ermini 1974b: 52, Tav. XVI, n. 31; da ultimo Pensabene 2015: 406 e 409, fig. 591a-b.

${ }^{32}$ Un destino analogo fu riservato alle lastre ritagliate dai plutei di Santa Maria in Domnica delle quali il verso fu verosimilmente approntato per il reimpiego nel pavimento moderno, v. RANUCCI 2003: 219.

${ }^{33}$ Pani Ermini 1974a: Tav. XXVII, n. 61 (cornice inferiore) e Tav. XXVIII, n. 63.
} 
La peculiare modanatura delle cornici costituisce un vero e proprio distintivo dell'officina pascaliana. Interpretando in modo originale la cornice delle lastre di VI secolo, i lapicidi e gli scultori di Pasquale I si allinearono infatti a una più generale ripresa della forma e della decorazione dei plutei paleocristiani (quali per es. i plutei della recinzione di papa Giovanni II a San Clemente), ${ }^{34}$ avviata nella basilica di San Pietro al tempo di Gregorio III (731-741) e proseguita al tempo di Leone III (795-816). ${ }^{35}$

Il sistema complesso della cornice consta di una larga fascia perimetrale e di tre listelli "a dente" digradanti verso l'esterno. ${ }^{36}$ Purtroppo lo stato frammentario dei plutei di Santa Prassede permette di ricomporre solo idealmente l'effetto prospettico indotto dalle cornici sull'ornato delle specchiature. Con l'aiuto delle illustrazioni, proverò a evocare questo effetto con le parole.

Oltre la fascia piatta che corre ai margini, il marcato contrasto luce-ombra dei tre listelli "a dente", crea un perimetro concentrico che stringe e delimita il campo decorato con motivi geometrici, vegetali e a intreccio. In altre parole, il profilo della cornice bilancia e per così contiene le esuberanti specchiature. ${ }^{37}$ Almeno in alcuni esemplari, esse ripropongono la geometria simbolica delle recinzioni di VI secolo - rombi e quadrati intrecciati con croci inscritte - ma, in modo inedito, il piano di fondo fiorisce di corolle, elici, croci gigliate e nodi a tre punte che immettono una vitalità nuova negli schemi paleocristiani.

Per designare questi "rilievi", che sono in realtà ottenuti mediante l'abbassamento del piano di fondo, occorrerebbe coniare un termine $a d$ hoc; in effetti, è come se le forme geometriche o vegetali affiorassero al piano di sculturazione che si mantiene a una quota lievemente più bassa rispetto alla cornice perimentrale. Se l'invenzione non è nuova, qui ha qualcosa di sorprendente: quella che Angiola Maria Romanini definiva «l'astratta bidimensionalità dell'immagine tardoantica, paleocristiana, bizantina», ${ }^{38}$ nelle specchiature dei plutei di Pasquale I, è mediata classicamente dalla cornice che non inquadra delle scene figurate - si rammentino i rilievi della porta lignea di Santa Sabina -, ma delle composizioni squisitamente astratte, anche quando è protagonista il tralcio fogliato. (fig. 8) Questa funzione "prospettica" della cornice ha d'altra parte una finalità perchè, per veicolare il suo messaggio simbolico, lo specchio decorato doveva essere osservato non solo nel suo insieme e a una certa distanza, ma anche nella relazione tra le distinte componenti strutturali del setto presbiteriale (pluteo-pilastrino-pluteo).

\footnotetext{
${ }^{34}$ Sulle varianti della modanatura (tipologie $a-e$ ) rilevate recto-verso nei plutei di San Clemente v. nel dettaglio Barsanti, Guiglia Guidobaldi 1992: 80-81.

${ }^{35}$ BALLARDini 2008: 233-239.

${ }^{36} \mathrm{Nel}$ caso di un frammento di pluteo spartito in due specchiature (Corpus CISAM, VII, 1, n. 62), interna ai listelli "a dente" è scolpita una fascia un poco più larga, cfr. la tipologia $a$ delle modanature dei plutei di San Clemente (per. es. Corpus CISAM, VII, 7, n. 50A).

${ }^{37}$ Di un effetto bianco/nero del rilievo che trionfa nella scultura di età pascaliana ha parlato Gianclaudio Macchiarella, v. MaCCHIARELla 1976b: 297.

${ }^{38}$ RoMANINI 1971: 439.
} 
A questo proposito, tornano alla mente alcune osservazioni di Leslie Brubaker che, scrivendo della retorica della comunicazione visuale, nota come la decorazione "aniconica" (sia essa utilizzata nella partitura di un manoscritto liturgico o nello spazio architettonico di una chiesa), «directs the viewer to understand what is seen in a particular way» e a coglierne il sistema di gerarchie ${ }^{39}$ Come i rivestimenti delle parti inferiori dei muri in opus sectile o in tessuto (veri o dipinti), gli stucchi geometrici o floreali, gli ornamenti dei portali o dei sottarchi, così anche $\mathrm{i}$ recinti presbiteriali a decoro astratto segnalavano al devoto o al chierico quale dovesse essere il suo posto in quello spazio. Per usare le parole della Brubaker «Aniconic decoration keeps the church visitor firmly in place» e «...like other non-narrative phenomena such as music or scent, it is a powerful manipulator of perceptions, establishing a framework that silently structures meaning and a mode of understanding beyond words». ${ }^{40}$

Accanto agli schemi decorativi di derivazione paleocristiana, i plutei di Santa Prassede accolgono anche motivi geometrici di nastri viminei che si annodano in maglie quadrate con croci inscritte; oppure in grandi cappi a due punte o ancora in composizioni di maglie circolari che racchiudono delle rosette a elica. Si tratta di variazioni del Flechtwerkdecor che certo a Santa Prassede sono oggi penalizzate dalla dispersione dei materiali. È però significativo che, a fronte di una affinità esecutiva, il repertorio a intreccio superstite nei frammenti delle altre basiliche pascaliane segua altri schemi. Se il rapporto tra conservato e perduto costringe alla prudenza, il confronto tra i frammenti scultorei di Santa Prassede, di Santa Cecilia e di Santa Maria in Domnica induce a pensare che i maestri scultori di Pasquale I, almeno per il Flechtwerkdecor, avessero messo a punto cartoni ad hoc per le diverse basiliche. (fig. 9)

Un accenno richiede, infine, un motivo compositivo attestato a Santa Prassede (e anche a Santa Cecilia), che è assai noto a chi si occupi di scultura alto medievale e che Rudolf Kautzsch nel 1939 definì Korbboden ${ }^{41} \mathrm{Nel}$ tentativo di dare un nome alle cose, con l'espressione "a fondo di cesto" lo studioso tedesco cercò una qualche «relazione di similarità» tra il motivo - che è invece una composizione geometrica puramente astratta - e «un oggetto del mondo»: ${ }^{42}$ gli sembrò di trovarla nella tessitura dei vimini attorno all'innesto a croce dei rametti di salice che è l'elemento strutturale della base di un cesto. Fabio Betti ha opportunamente preferito all'impropria definizione di Kautzsch, una lettura di carattere «cosmologico-cosmografico» della composizione geometrica proposta da Victor H. Elbern (1983), che considerò il motivo un'originale creazione "carolingia". ${ }^{43}$ Secondo lo studioso tedesco, gli elementi geometrici del motivo - si veda nell'esemplare di Santa Prassede il quadrato sulla punta inscritto nel cerchio, intersecato a croce da tiranti di nastri intrecciati che si dipanano dalla rosetta al centro della composizione per riannodarsi al nastro del cerchio (fig. 10) - esprimerebbe una visione del mondo e insieme un'immagine di salvezza cristologica.

\footnotetext{
${ }^{39}$ BRUBAKER 2004: 574.

${ }^{40}$ Brubaker 2004: 577 e 589.

${ }^{41}$ Kautzsch 1939: 9-12.

${ }^{42}$ Casartelli Novelli 1992: 535.

${ }^{43}$ Betti 1995: 131 e Elbern 1983: 21-22.
} 
Se si può essere d'accordo con Elbern sul natura astratta del motivo e sul messaggio cosmologico e cristologico che esso veicola, la marca schiettamente "carolingia" dell'invenzione chiede di riconsiderare la genesi del motivo che trova consonanza con antichi schemi geometrici di concezione non meno sofisticata. (fig. 11)

Da ultimo, in un nuovo studio ricostruttivo della barriera presbiteriale di San Benedetto di Mals (Val Venosta), Katrin Roth-Rubi, preferendo l'espressione Kreis-Raute-Kreutz-Motiv più aderente alla natura astratta e simbolica del fuorviante Korbboden, ha attirato l'attenzione sull'accostamento nella barriera di San Benedetto, ma anche in altri contesti alto medievali, del motivo "cerchiorombo-croce" al tralcio con foglie polilobate contrapposte. ${ }^{44}$ A Santa Prassede il tralcio con foglie polilobate contrapposte è adattato con originalità (e un effetto astratto ma sorprendemente organico e animato) alla specchiatura di un pluteo che ha dimensioni analoghe a quelle del pluteo con il doppio motivo "cerchio-rombo-croce". Come osserva Roth-Rubi, la non casualità dell'accostamento è provata dalla occorrenza dei due motivi nella lastra monolitica di Niederzell (Reichenau) e nel pluteo spartito in due specchiature, oggi nella galleria absidale esterna di Santa Maria e Donato a Murano. ${ }^{45}$ (fig. 12) La compresenza dei due motivi è stata inoltre accertata nel centro Italia a Nepi, nell'abbaziale di Sant'Elia (Corpus CISAM, VIII, nn. 164;165;173); nel Duomo di Ferentino (Corpus CISAM, XI, nn. 5; 23; 38; 40) e nella chiesa monastica di San Silvestro al Soratte (Corpus CISAM, VIII, nn. 142; 145; 143). In particolare, quest'ultima chiesa conserva reimpiegati in un gradino di accesso al podio absidale, un pilastrino con tralcio a foglie polilobate contrapposte e, nel fianco sinistro dell'altare romanico, un magnifico esempio del motivo "cerchio-rombo-croce" scolpito su una lastra di cipollino. ${ }^{46} \grave{E}$ naturale pensare che 1>accostamento dei due motivi stringesse in un nodo sintattico l'emblema cristologico e l'Albero della vita (nella forma di un tralcio in perenne crescita), proprio in virtù del vincolo strutturale che legava le distinte componenti del setto presbiteriale (pluteo-pilastrino-pluteo).

Dei frammenti scultorei, grandi e piccoli che sono pervenuti a Santa Prassede, la maggior parte presenta gradi variabili di consunzione della superfice e solo in un paio di casi ha senso fare delle osservazioni sulla tecnica esecutiva. ${ }^{47}$ (cfr. figg. 7 e 13)

È bene rammentare che quasi tutte le pietre superstiti sono di marmo cipollino (marmor carystium). L'omogeneità del materiale e le dimensioni considerevoli delle lastre lavorate nell'officina di Santa Prassede sono di per sè un indice delle possibilità economiche del committente e, anche se la scultura alto medievale è mediamente di rapida esecuzione, i lapicidi e gli scultori di Pasquale I dimostrarono

\footnotetext{
${ }^{44}$ Roth-Rubi 2015a: 95-105.

${ }^{45}$ Roth-Rubi 2015a: 103-108 e per il pluteo di Santa Maria e Donato di Murano, Ibsen 2008: 176-177 e fig. 35.

${ }^{46}$ La presenza di marmo greco al Soratte (un unicum tra i frammenti alto medievali pervenuti in quest'area) è un indizio significativo della provenienza dall'Urbe della pietra e degli scultori che, con singolare abilità, la decorarono.

47 Una prima osservazione tecnica dei frammenti pascaliani di Santa Prassede è stata diretta da Gianclaudio Macchiarella, v. Macchiarella 1976b: 282-284.
} 
cura e capacità nel valorizzare l'eccellente qualità del marmo che ebbero a disposizione. A tale riguardo (e come ipotesi di lavoro), c'è da chiedersi se l'assenza di tracce di una rifinitura colorata delle superfici non debba essere interpretata come un'effettiva mancanza all'origine. L'impiego quasi esclusivo del marmo cipollino, qui in una dominante chiara con belle venature grigio-blu, potrebbe in effetti rispecchiare una scelta di gusto del committente, alla quale seppero corrispondere le maestranze, abili nell'usare le variazioni cromatiche del marmo caristio come potenziamento espressivo delle forme scolpite.(cfr. fig. 8)

Sembra avvalorare questa ipotesi, la padronanza della tecnica degli scultori che sulla pietra nuda produssero degli effetti luce-ombra assai raffinati, si veda p.e. la modulata graduazione (in spessore e in profodità) delle pale delle rosette a elica, a loro volta inscritte in diametri quasi perfetti e dal profilo "a dente";(fig. 13) oppure l'inclinazione costante con la quale la gradina lavora sulla superficie dei listelli della cornice, ottenendo un effetto ruvido e opaco che reagisce con la superficie piatta e discretamente polita della fascia marginale. (fig. 14)

\section{Ricomporre i pezzi}

Il destino frammentario ed erratico subito dalla scultura alto medievale (in larga misura scultura per l'arredo liturgico) è uno degli ostacoli principali alla comprensione e al corretto inquadramento cronologico del fenomeno. Accostarsi a un frammento fuori contesto è infatti come giungere a Finisterre: in scultura, come in pittura, il frammento erratico è un "limite" oltre il quale (non di rado) si smarrisce il significato funzionale ed espressivo che l'uomo ha impresso all'artefatto. Il piccolo arcipelago di frammenti di Santa Prassede (comprensivo della scultura architettonica di San Zenone), offre l'occasione di cimentarsi "nel contesto" con una pratica artistica che è strettamente vincolata alla forma e alla funzione di un edificio di culto alto medievale, ma chiede altresì di superare i confini di un esclusivo studio tipologico dei motivi scultorei.

In effetti, l'indagine intorno alla formalizzazione del linguaggio della scultura alto medievale perde aderenza se non procede insieme con la conoscenza delle caratteristiche funzionali degli artefatti in pietra; se allo studio dello stile non unisce la comprensione delle tecniche e se non riconosce che gli standards qualitativi, alti o bassi che siano, non identificano lo stile di un periodo, ma un'operatività che, di volta in volta, si adegua alle richieste (e alle possibilità materiali e culturali) di una società complessa: un dato quest'ultimo che, nella Roma dei papi e nel Patrimonium Sancti Petri, invita ad aprirsi a una visione più articolata delle committenze in campo.

Tutto questo richiede un lavoro paziente e sperimentale, tal quale intrapresero, alla fine del XIX secolo, Raffaele Cattaneo e Ferdinando Mazzanti. ${ }^{48}$

\footnotetext{
${ }^{48} \mathrm{Si}$ veda la tavola di Ferdinando Mazzanti con alcuni rilievi alto medievali di San Zenone pubblicata da GHISU,
} RAIMONDI 2008: fig. 21. 
È davvero una fortuna che di tanto in tanto ci si imbatta in qualche "pietra miliare" che apre alla comprensione e fissa un punto di riferimento: ${ }^{49}$ vorrei ricordarne almeno una, per la parentela che mostra con le pietre di Pasquale I.

Conservata a Giulianello di Cori presso il Palazzo Salviati, la pietra della quale ignoriamo il contesto di provenienza è stata pubblicata da Domenico Palombi e di nuovo da Fabio Betti. ${ }^{50} \mathrm{Per}$ quanto erratico, questo frammento è paragonabile a un filamento di DNA che custodisce in sè una incredibile quantità di informazioni. ${ }^{51}$

La pietra, spezzata su ogni margine, è scolpita su entrambe le facce.(figg. 15-16) Un lato conserva intatta una tabula inscriptionis di eccellente fattura (si vedano la rigatura tracciata a punta sulla superficie polita; la sezione a cuneo del corpo delle lettere; gli apici e i puncti distinguentes a triangolo). Il significato del testo inscritto è esplicito quanto basta per datare i rilievi al pontificato di Leone III (795-816). ${ }^{52}$ L'eccezionalità del monumento, insieme scultoreo ed epigrafico, sta nella scelta dei motivi che ornano il recto e il verso. A circondare la tabella inscriptionis, sul recto della pietra, si dispone infatti un tralcio di foglie polilobate contrapposte che, nel trattamento del nastro vimineo e nella memoria irrigidita del tralcio a cornucopia, ha molto in comune con l'ornamento del noto pluteo di Santa Prassede..$^{53}$ La sorpresa maggiore è però riservata dal "lato B", dove il nastro bisolcato offre un'inedita interpretazione del tralcio con uva e pampini che discende, in linea diretta, dal raffinato repertorio scultoreo di età longobarda (v. Bobbio, lastra di Cumiano; Osimo, lastra di Vitaliano). In un periodo cruciale per il riassetto delle officine scultoree a Roma e nel Patrimonium Sancti Petri, il frammento di Giulianello permette dunque di toccare con mano la convergenza - in un ambiente papale - di lapicidi e di scultori eredi diretti di una tradizione esercitata e affinata per la committenza longobarda del più alto rango. ${ }^{54}$

Il recto dalla pietra, inoltre, con le sue anticipazioni degli esiti prodotti dalle officine scultoree pascaliane, conferma una tendenza già osservata nei mosaici di Leone III (Triclinio Lateranense e Santi Nereo e Achilleo), tendenza che dimostra come la riorganizzazione di maestranze capaci

\footnotetext{
${ }^{49} \mathrm{Si}$ veda, da ultimo, il censimento ragionato dei frammenti di scultura alto medievali databili avviato da Roтн-RUBI 2015b (Textband): 11-44.

${ }^{50}$ Palombi 2004: 30 e 33-35, figg. 11a e 11b e Betтi 2014: 141-142 e fig. 114.

${ }^{51}$ Con il collega Fabio Betti, devo un vivo ringraziamento alla signora Clara Sbardella per averci accolto a Palazzo Salviati e consentito di esaminare e di fotografare il frammento (4 novembre 2016).

${ }^{52}$ Divisa in due parti l'iscrizione oltre al riferimento cronologico Tempo/ribus / D(omi)ni Leo/ni Tertii / Papae (parte sinistra) comprende un'iscrizione enigmatica introdotta dal signum crucis (parte destra) che richiede un riesame o almeno «ulteriori approfondimenti» v. PANI 2004: 34.

${ }^{53}$ La superficie del pluteo di Santa Prassede è consunta e l'aspetto morbidamnete arrotondato del tralcio fogliato non corrisponde allo stato originario; rispetto al frammento di Giulianello, i lobi delle foglie, sono incise lungo i margini, ma non sono scavate a cuneo. Le incisioni a Kerbschnitt nel pluteo di Santa Prassede interessano solo (e non sempre) lo snodo del tralcio.

${ }^{54}$ La compresenza di tradizioni diverse può forse ravvisarsi anche nella variazione di modulo dei caratteri della doppia iscrizione: un modulo alto e compresso, quello della formula cronologica e un modulo spiccatamente quadrato ed "epigrafico" quello dell'iscrizione enigmatica.
} 
di riappropriarsi di tecniche antiche e di formalizzare linguaggi nuovi o rinnovati, fosse avvenuta, con esiti eccellenti, ben prima dell'esuberante settennato di Pasquale I.

A Santa Prassede è in particolare la scultura architettonica del sacello di San Zenone a dimostralo. Al netto di alcuni restauri e dei rifacimenti che interessarono la nicchia dell'altare, i marmi scolpiti del sacello sono un formidabile "incunabolo" che attende un ulteriore scandaglio. Tra i marmi alto medievali di reimpiego nel San Zenone, mi limito a segnalare, in corrispondeza del varco di accesso, l'architrave modanato con cornice a battente (ornato con kyma, astragali e trecce a due capi) e un frammento monolitico di stipite (il destro), ridotto a misura della porticina, ma ancora completo del profondo montante a sostegno dell'imbotto (Corpus CISAM, VII, 1, n. 90 e n. 88).(figg. 17-18)

Il raffinato lavoro a intreccio che orna lo stipite - un'invenzione tessile inserita in modo movimentato e quasi elastico nello specchio decorato $-^{55}$ parla dell'abilità dei suoi artefici e si esprime con un Flechtwerkdecor che fu apprezzato e imitato dagli scultori formatisi nelle officine di Pasquale I. ${ }^{56}$ L'adattamento dei pezzi indica invece che il portale (o i portali) dal quale l'architrave e lo stipite furono smontati era di discrete dimensioni e, come ovvio, attesta il terminus ante quem per l'età dei frammenti (prima degli anni 817-824).

Infine, l'eccellente fattura di questi elementi architettonici, dimostra che le officine scultoree papali degli immediati predecessori di Pasquale I avevano già raggiunto un grado di distinzione. ${ }^{57}$

\footnotetext{
${ }^{55} \mathrm{Si}$ vedano a questo proposito, le riflessioni di b-Rubi su una serie di lavori a intreccio di analoga tipologia e fattura che la studiosa confronta con i rilievi di Coira, Rотн-Ruвi in stampa.

${ }^{56}$ Non a Santa Prassede, ma nell'arredo liturgico offerto dal papa ai Santi Giovanni e Paolo di Ferentino, v. RamieRI 1983: Tav. VI, n. 9; Tav. VII, n.11, Tav. XII, nn. 16-17, cfr. Ballardini 2008: 229-230 e Roth-RuBi 2015b: 30

${ }^{57}$ I livelli qualitativi della scultura si accompagnono anche a quelli delle epigrafi, BaLLARDINI 2008: 237-239; BALLARDINI 2010: 141-148 (Figg. 161-166).
} 


\section{Bibliografia}

Apollonj Ghetti, Bruno Maria, 1961. Santa Prassede, Roma, Marietti

Ballardini Antonella, 1999. "Dai gesta di Pasquale I secondo il Liber Pontificalis ai monumenta iconografici delle basiliche Romane di Santa Prassede, Santa Maria in Domnica e Santa Cecilia in Trastevere”, Archivio della Società romana di storia patria, 122: 5-68

-2008. "Scultura per l'arredo liturgico nella Roma di Pasquale I: tra modelli paleocristiani e Flechtwerk", Medioevo: arte e storia, X Convegno Internazionale di Studi, (Parma, 18-22 settembre 2007), A.C. Quintavalle (ed.), Milano-Parma, Electa, 2008: 225-246

-2009. "Da ornamento a monumento: la scultura altomedievale nella storiografia di secondo Ottocento", Medioevo: immagine e memoria, XI Convegno Internazionale di Studi, (Parma, 2328 settembre 2008), A.C. Quintavalle (eds.), Milano-Parma, Electa: 109-126

-2010. "Scultura a Roma: standards qualitativi e committenza (VIII secolo) ", L'VIII secolo: un secolo inquieto, Convegno Internazionale di Studi, (Cividale del Friuli, 4-7 dicembre 2008), V. Pace (ed.), Cividale del Friuli, Comune di Cividale del Friuli, 2010: 141-148

-2013. "Raffaele Cattaneo, Venezia, San Marco", La storia dell'arte a Venezia ieri e oggi: duecento anni di studi, Atti del Convegno di studi (Venezia, 5-6 novembre 2012), X. Barral i Altet, M. Gottardi (eds.), Ateneo Veneto, 200, iii s. 12/I: 149-167

-2016. "Selvatico e gli studi dedicati a S. Marco", Pietro Selvatico e il rinnovamento delle arti nell'Italia dell'Ottocento, Atti del convegno (Venezia, 22-23 ottobre 2013), A. Auf der Heyde, M. Visentin, F. Castellani (eds.), Pisa, Edizioni della Normale-Venezia, Istituto Veneto di Scienze, Lettere ed Arti: $331-350$

Barsanti, Claudia, Flaminio, Roberta, Guiglia, Alessandra, 2015. Corpus della scultura altomedievale, VII, La Diocesi di Roma, 7, La III Regione Ecclesiastica, Spoleto CISAM

Barsanti, Claudia, Guiglia Guidobaldi, Alessandra, 1992. "Catalogo", San Clemente. La scultura del VI secolo, F. Guidobaldi, C. Barsanti, A. Guiglia Guidobaldi (eds.), Romae apud S. Clementem, Collegio di San Clemente: 79-83

Bellanca, Calogero, 1999, La basilica di Santa Sabina e gli interventi di Antonio Muñoz, Roma, Convento Santa Sabina

-2003 Antonio Muñoz. La politica di tutela dei monumenti di Roma durante il Governatorato, Roma, «L'Erma» di Bretschneider 
BetTi, Fabio, 1995, "Il transetto protocarolingio della cattedrale di Ascoli Piceno. La documentazione del restauro ottocentesco attraverso gli appunti e i disegni di Giulio Gabrielli”, Arte medievale, 2.Ser. 9, 2: 119-139

-2008, "Inedite testimonianze grafiche dell'attività di Antonio Muñoz conservate presso il Museo di Roma (Gabinetto comunale delle stampe)", Bollettino dei Musei Comunali di Roma, n.s., 22: 169-155

-2014, "Testimonianze scultoree carolinge a Cori e nel suo circondario", Una strada nel Medioevo. La via Appia da Roma a Terracina, M. Righetti (eds.), Roma Campisano Editore: $139-150$

Bianconi, Daniele, 2015, "Libri e letture di corte a Bisanzio da Costantino il Grande all'ascesa di Alessio I Comneno", Le corti nell'alto medioevo, LXXII Settimana di studio del Centro Italiano di Studi sull'Alto Medioevo (24-29 aprile 2014), Spoleto, CISAM: 767-815, Tavv. I-VIII

Brubabaker, Leslie, 2004. "Aniconic Decoration in the christian World (6th-11th century): East and West", Cristianità d'occidente e cristianità d'oriente (secoli VI-XI), LI Settimana di studio della Fondazione Centro Italiano di Studi sull'alto medioevo (Spoleto, 24-30 aprile 2003), Spoleto CISAM, 1: 573-590, Tavv. I-X

CAPERna, Maurizio, 1999. La Basilica di Santa Prassede. Il significato della vicenda architettonica, Roma, Monaci Benedettini Vallombrosani

-2014. La Basilica di Santa Prassede. Il significato della vicenda architettonica, nuova ed., riv. e aggiornata, Roma, Edizioni Quasar

Casartelli Novelli, Silvana, 1992. "Committenza e produzione 'scultorea bassa", Committenti e produzione artistico-letteraria nell'alto medioevo occidentale, XXXIX Settimana di studio del Centro Italiano di Studi sull'Alto Medioevo (4-10 aprile 1991), Spoleto, CISAM: 531-567, Tavv. I-LVI

Cattaneo, Raffaele, 1889. L'architettura in Italia dal VI secolo al Mille circa, Ricerche storicocritiche, Venezia Ongania

Ciampini, Giovanni Giustino, 1699. Vetera Monimenta, In quibus praecipue Musiva Opera Sacrarum, Profanarumque Aedium Structura, Ac nonnulli antiqui Ritus, Dißertationibus, Iconibusque illustrantur, Romae, Komarek 
Davanzati, Benigno, 1725. Notizie al pellegrino della Basilica di Santa Prassede, Roma, Antonio de’ Rossi

DE BlaAuw, Sible, 2006. "Innovazioni nello spazio di culto fra basso Medioevo e Cinquecento", la perdita dell'orientamento liturgico e la liberazione della Lo spazio e il culto, navata relazioni tra edificio ecclesiale e uso liturgico dal XV al XVI secolo ; [atti delle giornate di studio, 27 - 28 marzo 2003], Jörg Stabenow (ed.), Venezia, Marsilio: 25-51

ElBern, Victor. H., 1983. "Bildstruktur-Sinnzeichen-Bildaussage. Zusammenfassende Studien zur unfigurlichen Ikonographie im frühen Mittelalter”, Arte Medievale, 1: 17-37

Emerick, Judson J., 2001. "Focusing on the Celebrant: The Column Display inside Santa Prassede", Atti del colloquio internazionale Arredi di culto e disposizioni liturgiche a Roma da Costantino a Sisto IV (Istituto Olandese a Roma, 3-4 dicembre 1999), Mededelingen van het Nederlans Instutuut te Rome, 59, pp. 129-159

FaVREAU, Robert, 1992. "Le commanditaires dans les inscriptions du haut moyen age occidental", Committenti e produzione artistico-letteraria nell' alto medioevo occidentale, XXXIX Settimana di studio del Centro Italiano di Studi sull'Alto Medioevo (Spoleto, 4-10 aprile 1991), Spoleto, CISAM: 681-727.

-1997. Épigraphie médiévale, Turnhout, Brepols

Finocchio, Giuseppe, 2010. "Riflessioni sul pavimento presbiteriale della chiesa di S. Prassede", Atti del XV colloquio dell'Associazione Italiana per lo studio e la conservazione del mosaico (Aquileia, 4-7 febberaio 20009) C. Angelelli, C. Salvetti (eds.), Tivoli (Roma), Scripta Manent Edizioni: $305-314$

Ghisu, Sandra, Raimond, Elena 2008. "Ferdinando Mazzanti: figura e opera", Archivio della Società di Storia Patria, 131: 294-311

GianANDREA, Manuela, 2011. "Note sul perduto arredo liturgico di Santa Sabina all'Aventino nel corso del Medioevo", Rivista dell'Istituto Nazionale d'Archeologia e Storia dell'Arte, 3.Ser., 34,66: $151-164$

Guidobaldi, Federico, Guiglia Guidobaldi, Alessandra, 1983. Pavimenti marmorei di Roma dal IV al IX secolo, Roma, Pontificio Ist. di Archeologia Cristiana 
IBSEN, Monica, 2008, "L'arredo liturgico di Maguzzano. Contesti locali, irradiazioni sovraregionali, migrazioni”, Da fondazione privata a monastero del vescovo di Verona: Maguzzano (Lonato, BS) e la sua dipendenza di Soiano, G.P. Brogiolo, A. Chavarria, M. Ibsen (eds.), Archeologia Veneta, 29-30: 23-61

Kautzsch, Rudolf, 1939. "Die römische Schmuckkunst in Stein von 6. bis zun 10. Jahrhundert", Römisches Jahrbuch für Kunstgeschichte, 3: 3-73

Krautheimer, Richard, Corbett, Spencer 1971. Corpus basilicarum christianarum Romae, 3 : 235-262;

Macchiarella, Gianclaudio, 1976a. "Seminario sulla tecnica e il linguaggio della scultura a Roma tra VIII e IX secolo", Roma e l'età carolingia, Atti delle Giornate di Studio (3-8 maggio 1976), Istituto di Storia dell'arte dell'Università di Roma (ed.), Roma, Multigrafica Editrice: 267-288

-1976b. "Note sulla scultura in marmo a Roma tra VIII e IX secolo", Roma e l'età carolingia, Atti delle Giornate di Studio 3-8 maggio 1976, Istituto di Storia dell'arte dell'Università di Roma (ed.), Roma, Multigrafica Editrice: 288-299

MARANGONI, Giovanni, 1744. Delle cose gentilesche e profane trasportate ad uso, e adornamento delle chiese, Roma, Pagliarini

Melucco Vaccaro, Alessandra, 1974. Corpus della scultura altomedievale, VII, La Diocesi di Roma, 3, La II Regione Ecclesiastica, Spoleto CISAM.

Muñoz, Antonio, 1918. "Studii sulle basiliche romane di S. Sabina e di S. Prassede", Atti della Pontificia Accademia Romana di Archeologia. Dissertazioni, 2. Ser., XIII: 119-128

Noble, Thomas F.X., 2009. Images, iconoclasm, and the Carolingians, Philadelphia, Univ. of Pennsylvania Press

Palombi, Domenico, 2004. "Dall'antichità al medioevo", Julianum - Giuliano - Giulianello: materiali per la storia di un centro minore del Lazio meridionale, D. Palombi (ed.), s.l., Pontinia: 30-32

Pani Ermini, Letizia, 1974a. Corpus della scultura altomedievale, VII, La Diocesi di Roma, 1, La IV Regione Ecclesiastica, Spoleto, CISAM.

-1974b. Corpus della scultura altomedievale, VII, La Diocesi di Roma, 2, La Raccolta dei Fori Imperiali, Spoleto, CISAM. 
PANI, Gian Giacomo, 2004. “L'epigrafe di Leone III”, Julianum-Giuliano-Giulianello: materiali per la storia di un centro minore del Lazio meridionale, D. Palombi (ed.), s.1., Pontinia: 34

Panvinio, Onofrio, 1570, Le sette chiese Romane del R.P.F. Onofrio Panvinio Veronese, tradotte da Marco Antonio Lanfranchi, Roma, per gli eredi di Antonio Blado

Pensabene, Patrizio, 2015. Roma su Roma. Reimpiego architettonico, recupero dell'antico e trasformazioni urbane tra il III e il XIII secolo, Città del Vaticano, Pontificio Istituto di Archeologia Cristiana

RAmieri, Anna Maria, 1983. Corpus della scultura altomedievale, XI, La Diocesi di Ferentino, Spoleto CISAM.

RANuCCI, Cristina, 2003. "Elementi di arredo liturgico altomedievalein Santa Maria in Domnica. Rilettura complessiva dei materiali", Caelius I. Santa Maria in Domnica, San Tommaso in Formis e il Clivus Scauri, A. Englen (ed.), Roma, «L'Erma» di Bretschneider: 218-227

Raspi Serra, Joselita, 1974. Corpus della scultura altomedievale, VIII, La Diocesi dell'Alto Lazio, Bagnoregio - Bomarzo - Castro - Civita Castellana - Nepi - Orte - Sutri - Tuscania, Spoleto, CISAM.

Righetti, Marina, 2007. "Pasquale I e la fondazione carolingia", Santa Cecilia in Trastevere, C. La Bella, A. Lo Bianco, P. Marchetti, N. Parmegiani, S. Petrocchi, A. Pronti, D. Radeglia, M. Righetti (eds.), Roma, Palombi Editori: 78-81

Romanini, Angiola Maria, 1971. "Problemi di scultura e plastica altomedievali", Artigianato e tecnica nella società dell'ato medioevo occidentale, XVIII Settimana di studio del CISAM (2-8 aprile 1970), Spoleto 1971, Spoleto, CISAM: 425-467, Tavv. I-XLII

Rotн-Rubi, Katrin, 2015a. "Neu und doch nicht fremd. Zum Motiv eines unlängst in Mals entdeckten Pfostens mit Flechtwerkdekor und zur Schranke in St. Benedikt”, Antiquitates Tyrolenses. Festschrift für Hans Northdurfter, P. Gleirscher, L. Andergassen (eds.), Innsbruck, Universitätsverlag Wagner, 2015: 95-105

-2015b. Die frühe Marmorskulptur aus dem Kloster St. Johann in Müstair, 1. Textband, 2. Katalogband, Ostfildern, Thorbecke 2015 
Rотн-RuBi, Katrin, in stampa, "Die frühmittelalterliche Skulptur von Chur, Schänis, Mals und dem übrigen Vinschgau".

Russo, Eugenio, 2012. "Raffaele Cattaneo, Architekt, Kunsthistoriker", Personenlexikon zur Christilchen Archäologie. Forscher und Persönlichkeiten vom 16. bis zum 21. Jahrhundert, S. Heid, M. Dennert (eds.), 1: 291-292

Trinci Cecchelli, Margherita, 1976. Corpus della scultura altomedievale, VII, La Diocesi di Roma, 4, La I Regione Ecclesiastica, Spoleto, CISAM.
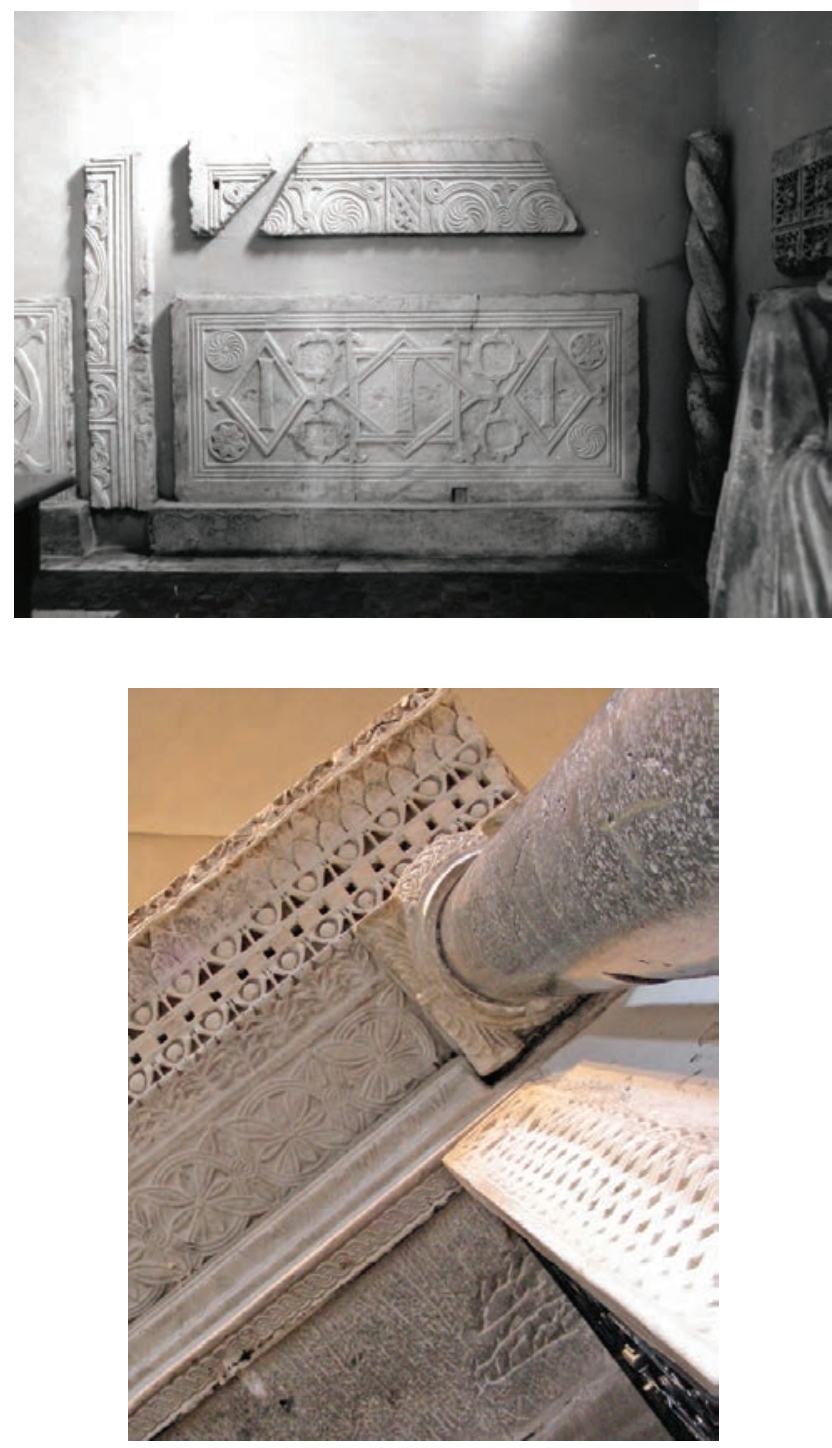

Fig. 1 Roma, Santa Prassede, pluteo e frammenti (da BALLARDINI 2008).
Fig. 2 Santa Prassede, sacello di San Zenone, reimpiegi e rilavorazioni (https://commons.wikimedia.org/wiki/ File\%3ASPrassedeSZenonePortale03) 

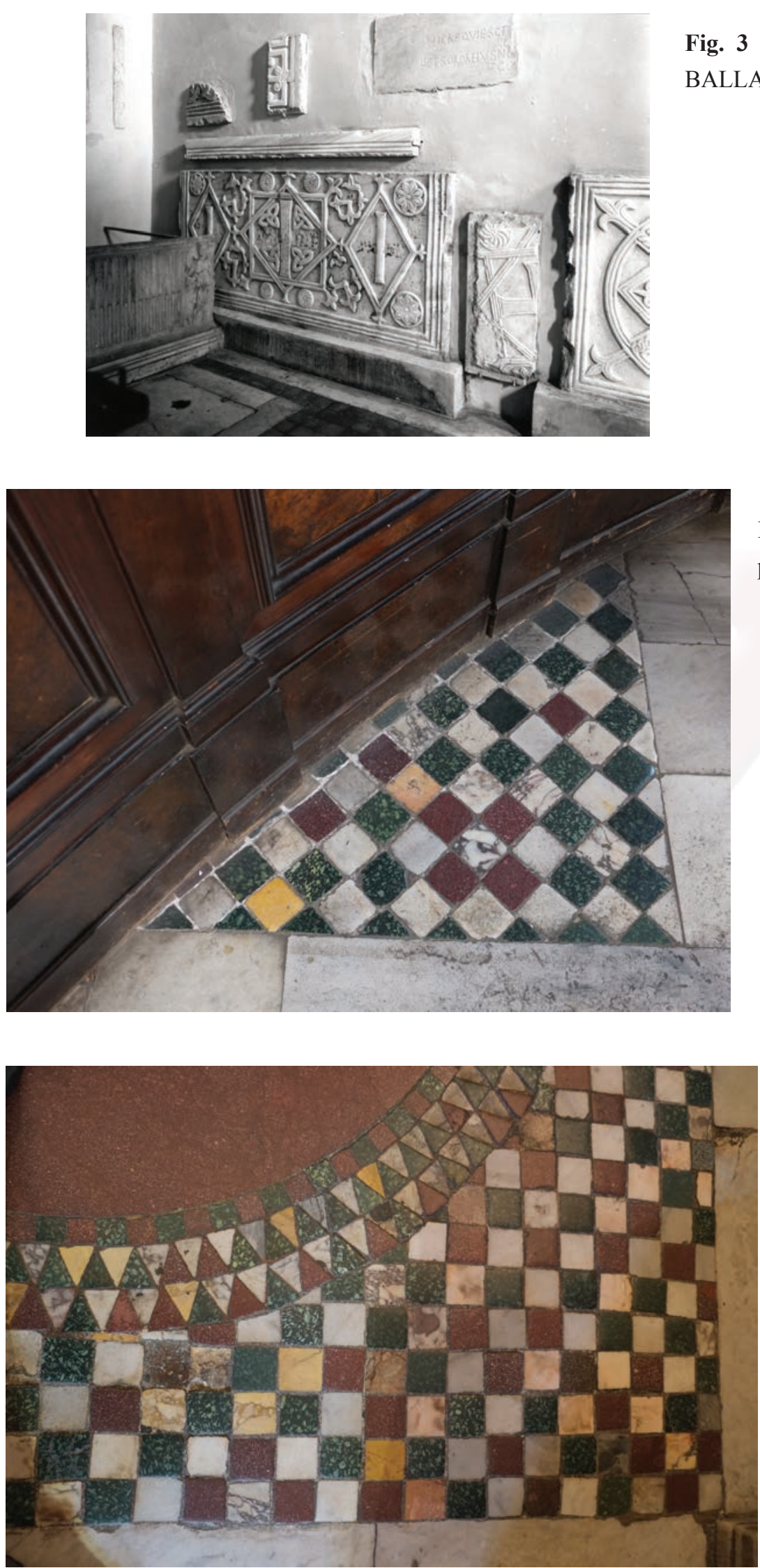

Fig. 3 Roma, Santa Prassede, pluteo e frammenti (da BALLARDINI 2008).

Fig. 4 Santa Prassede, sacello di San Zenone, particolare del pavimento (foto Autore).

Fig. 5 Santa Prassede, podio absidale, particolare del pavimento (foto Autore). 

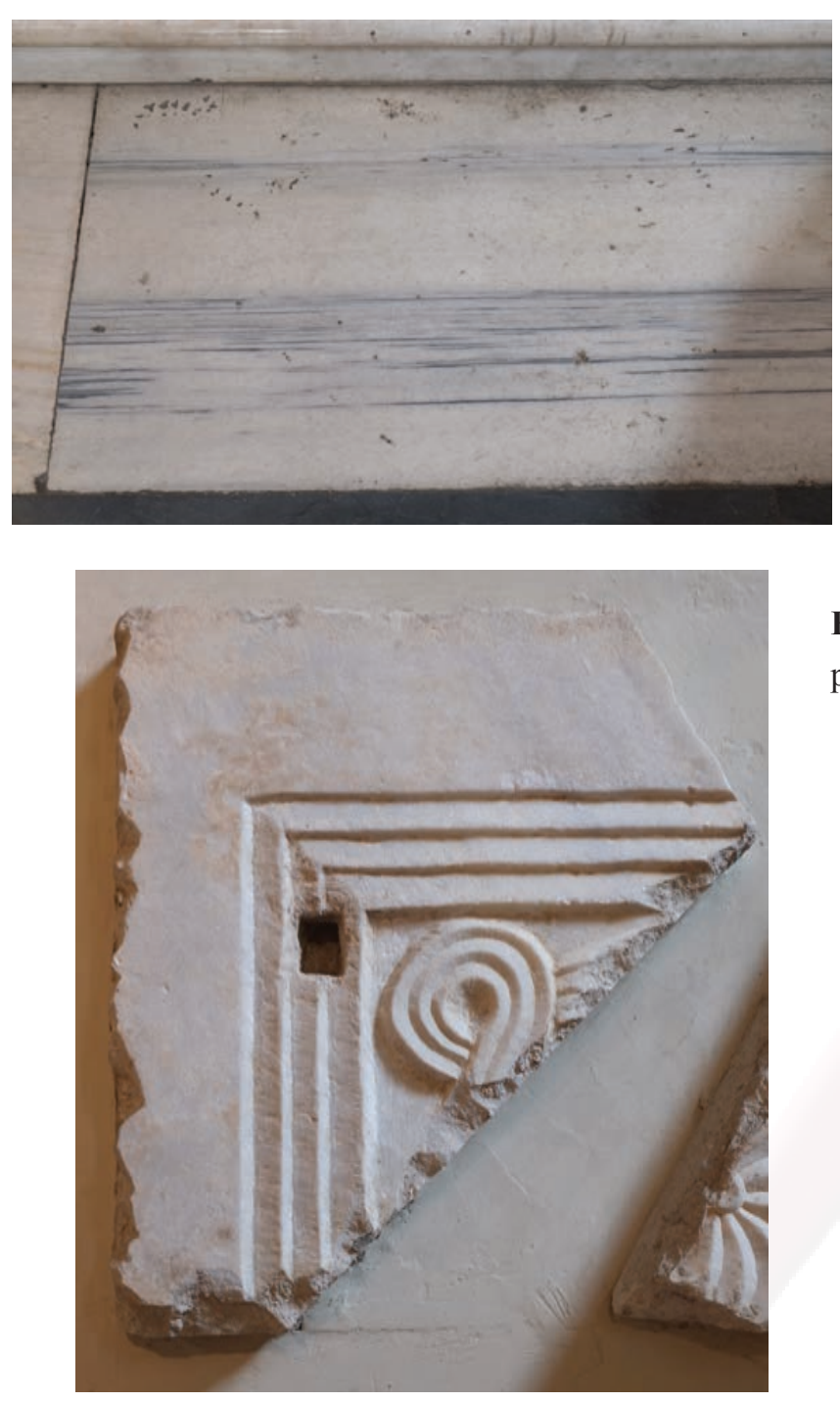

Fig. 6 Santa Prassede, podio absidale, lastra di cipollino con tracce di un decoro eraso (foto Autore).
Fig. 7 Roma, Santa Prassede, frammento di cornice di un pluteo (foto Autore).

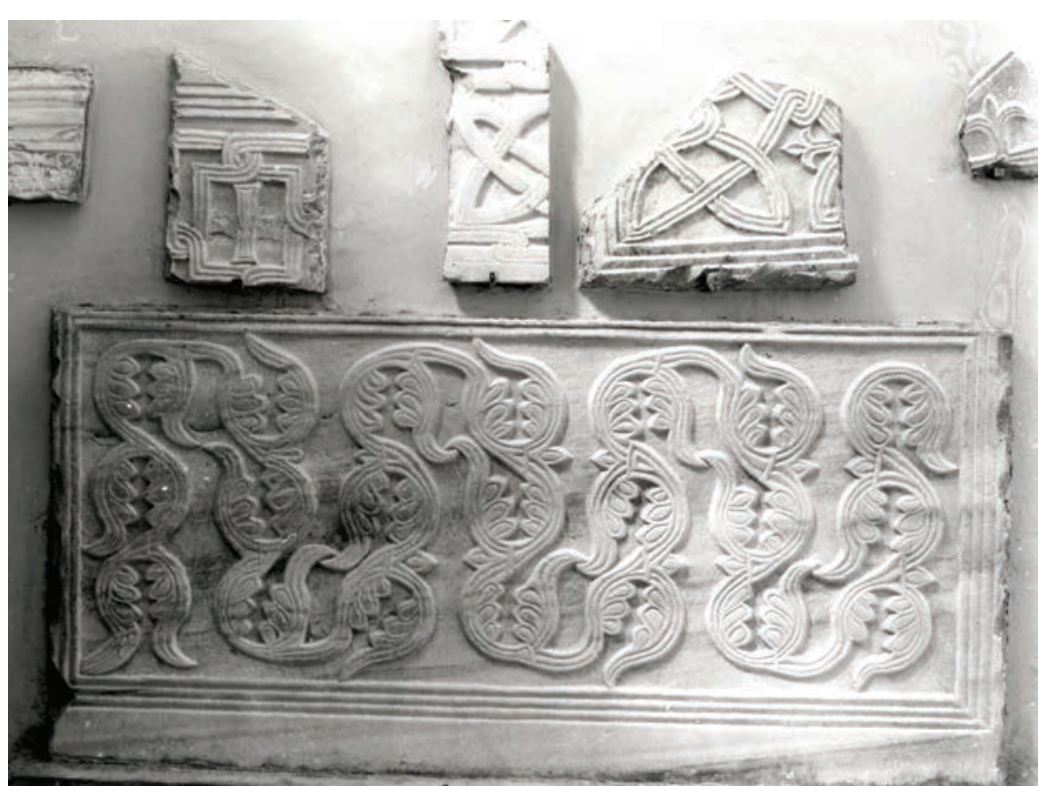

Fig. 8 Roma, Santa Prassede, pluteo con tralcio di foglie polilobate contrapposte (foto Autore). 


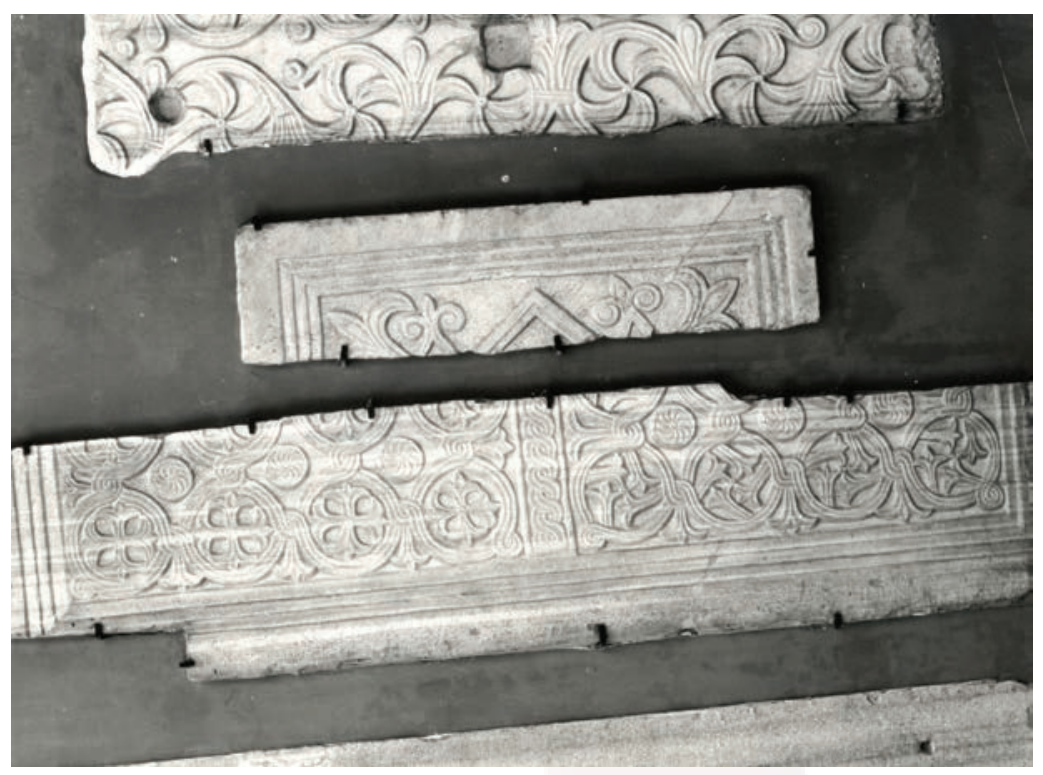

Fig. 9 Roma, Santa Cecilia in Trastevere, plutei frammentari (foto Autore).

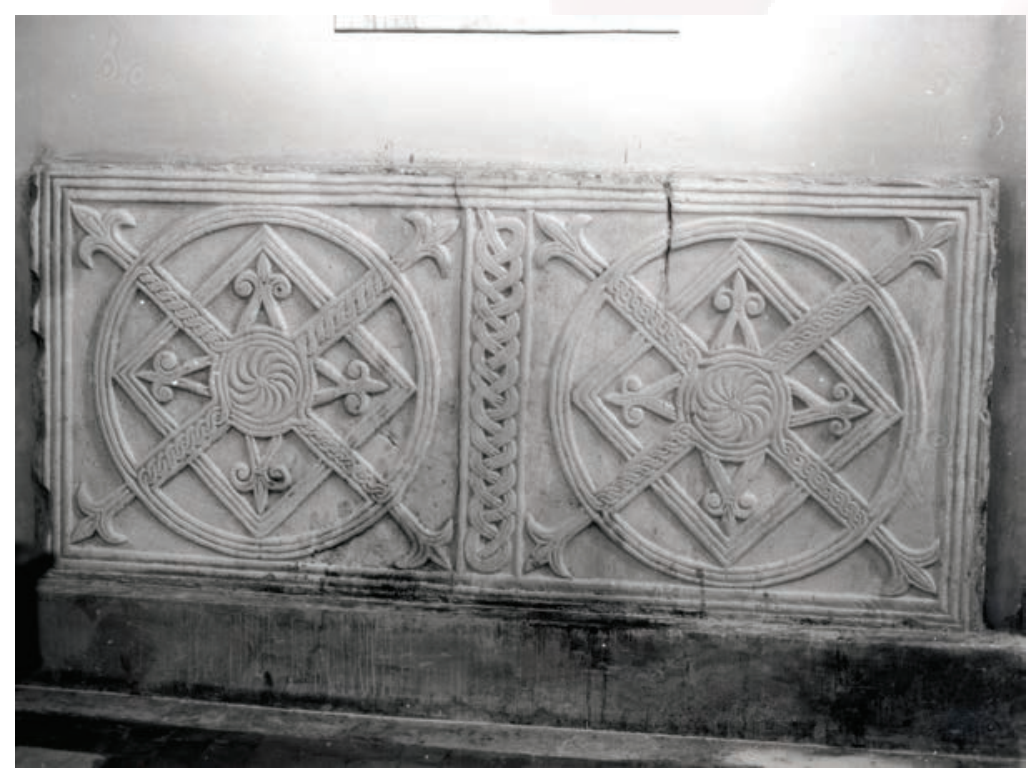

Fig. 10 Roma, Santa Prassede, pluteo con motivo "cerchio-rombo-croce" (foto Autore).

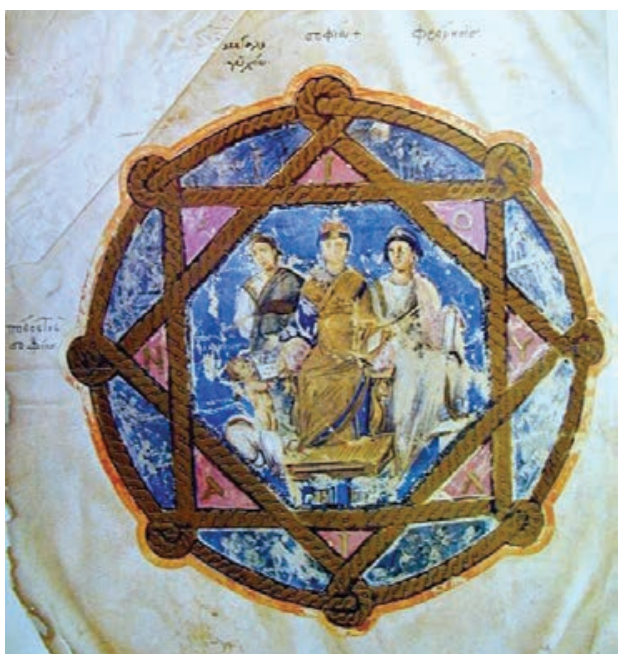

Fig. 11 Vienna, Österreichische Nationalbibliothek, Med. gr. 1, f. 6v, (in. VI sec.) (da BIANCONI 2015). 

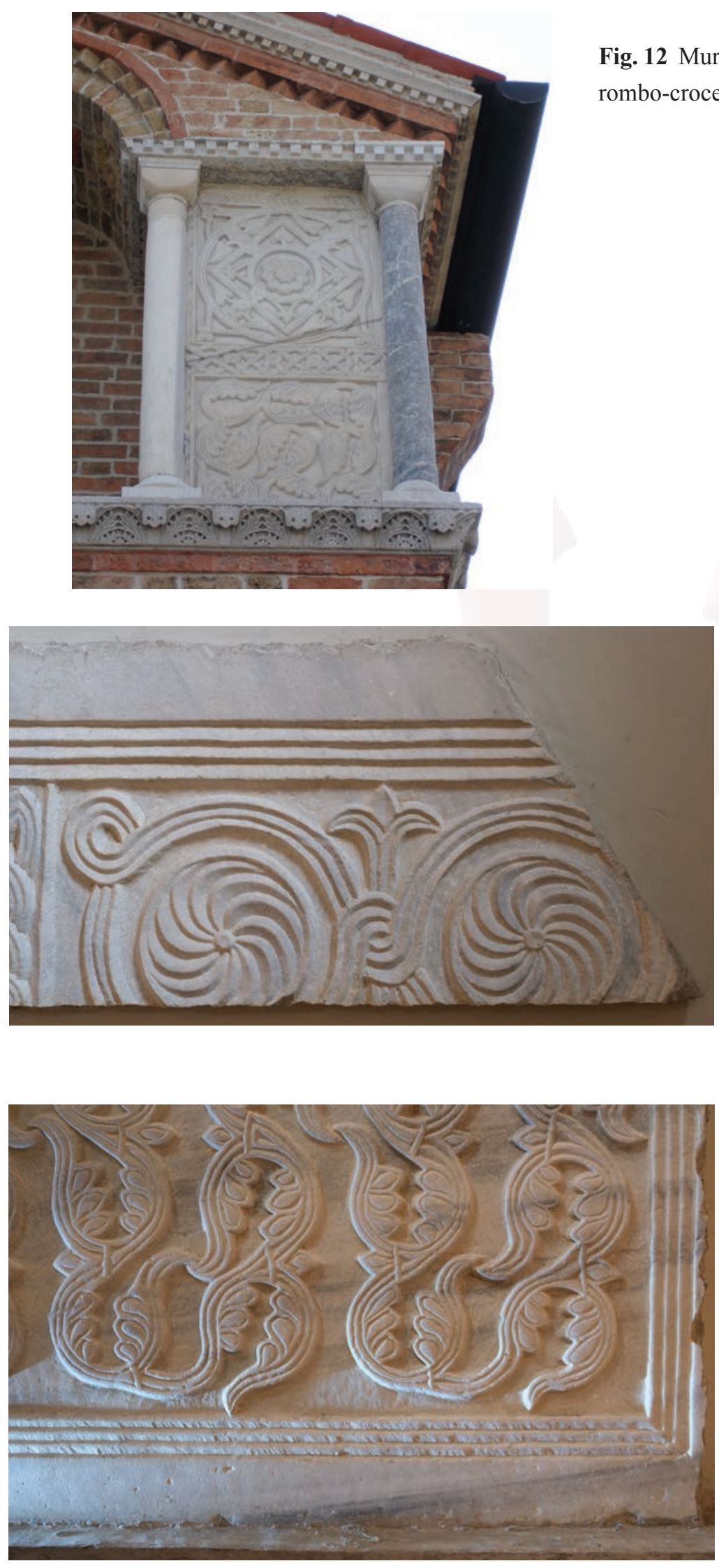

Fig. 12 Murano, Santa Maria e Donato, pluteo con motivo "cerchiorombo-croce" e con tralcio di foglie lobate (da IBSEN 2008).

Fig. 13 Roma, Santa Prassede, frammento di pluteo con nastro vimineo e rosette a elica (foto Autore).

Fig. 14 Roma, Santa Prassede, particolare del pluteo con tralcio di foglie polilobate contrapposte (foto Autore). 


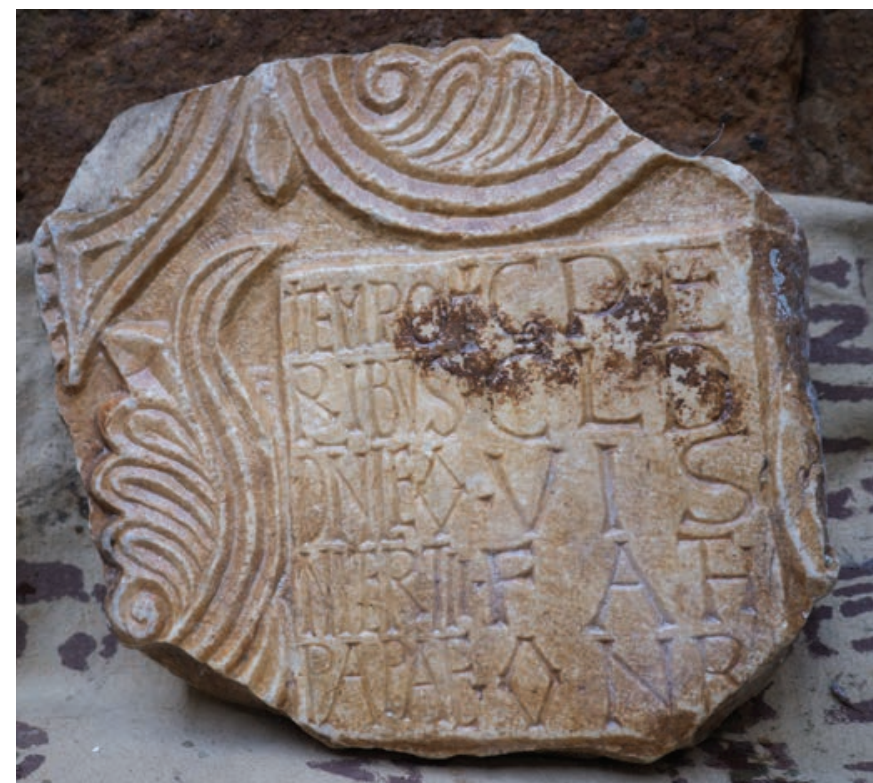

Fig. 15 Giulianello di Cori, Palazzo Salviati, frammento di lastra con iscrizione (recto) (foto Autore).

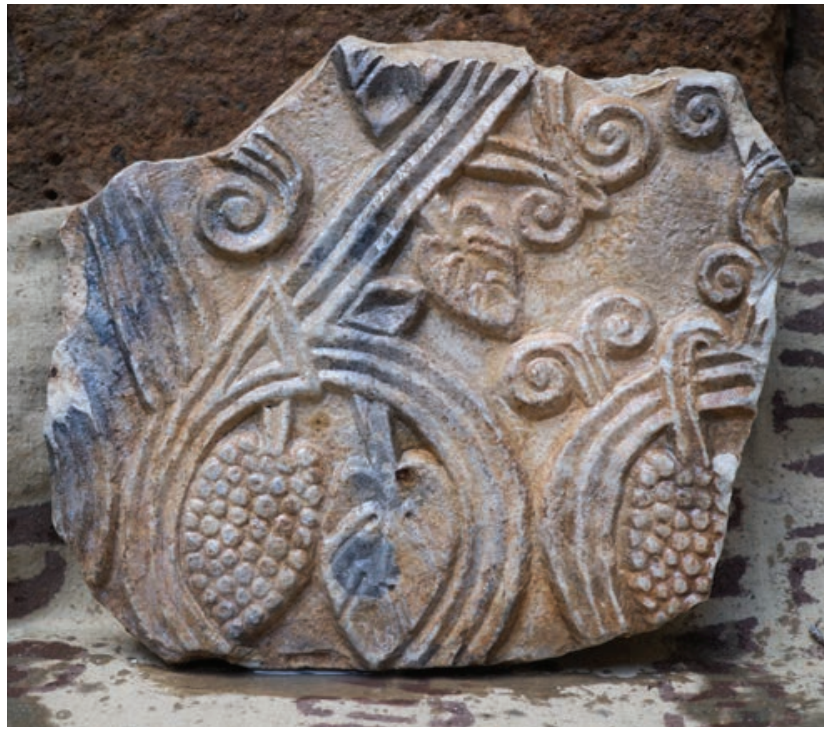

Fig. 16 Giulianello di Cori, Palazzo Salviati, frammento di lastra con iscrizione (verso) (foto Autore).

Fig. 17 Santa Prassede, sacello di San Zenone, particolare dell'architrave con kyma, astragali, e trecce a due capi (foto Autore).
Fig. 18 Santa Prassede, sacello di San Zenone, particolare dello stipide destro (foto Autore).

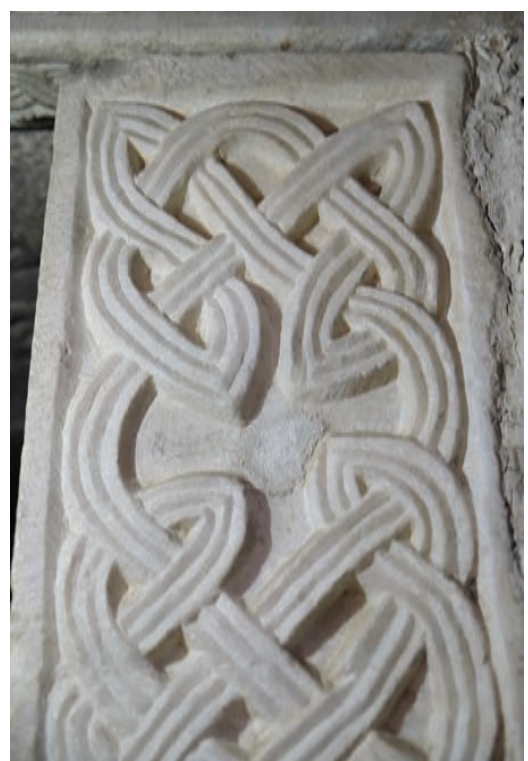

